\title{
袁亚湘：办中国最好的数学刊物
}

杨志华

\section{An interview with Ya-xiang Yuan: Run the best mathematical journal in China}

\section{Zhihua Yang}

doi: 10.1360/TB-2020-1293

袁亚湘, 中国科学院院士, 中国科学院数学与系统科 学研究院研究员. 现任全国政协常委、中国科协副主席、 国际工业与应用数学联合会主席, 发展中国家科学院院 士、巴西科学院通讯院士、美国数学会首届会士、美国工 业与应用数学学会会士. 主要从事运筹学研究并取得了系 统成果, 在信赖域法、拟牛顿法、非线性共轭梯度法等方 面作出了重要贡献. 袁亚湘1995年开始在《中国科学》发 表代表性研究成果, 1996年起担任《中国科学》编委, 2013 年起担任《中国科学: 数学》主编至今. 他以强烈的责任 感和饱满的热情积极推动期刊发展, 为提升期刊学术质量 和国际影响力作出了突出贡献.

在《中国科学》《科学通报》创刊70周年之际, 《中国 科学》杂志社专访袁亚湘主编. 在这次访谈中, 袁主编回 顾了他在《中国科学》发表的代表性文章, 追忆了他从《中 国科学》编委到《中国科学: 数学》主编期间, 履职尽责 推动刊物发展, 提升编委和作者参与度, 使得数学界越来 越多的人认可 《中国科学: 数学》是中国最好的数学刊物. 他以科学家强烈的使命担当, 呼吁改革数学教育和加强文 化自信, 并建言谋划刊物的长久发展.

\section{钻研难题，在《中国科学》发表代表性成果}

您在《中国科学》发表过很多文章, 还记得第一篇 吗？当时是怎么决定向《中国科学》投稿的？

袁亚湘: 我发表在《中国科学》的第一篇文章是 1994 年投稿、1995年发表的关于 DFP算法收敛性的文章 “Convergence of DFP algorithm”. DFP是世界上第一个拟 Newton算法, DFP对于凸函数是否收玫, 是优化领域最重 要的两个问题之一. 我的这篇文章, 在某些条件和假定下, 证明了DFP的收玫性, 虽然没有彻底解决这个问题, 但作 为一项阶段性的工作, 还是取得了不错的结果. 20 世纪 90 年代初我从德国回国之后, 因为我的研究工作比较受大家 认可, 所以杨乐先生希望我的工作能在《中国科学》有所 体现, 应杨先生之邀, 我把这篇文章投到了《中国科学》. 从发表这篇文章至今已有二十多年, 这个问题基本上还是

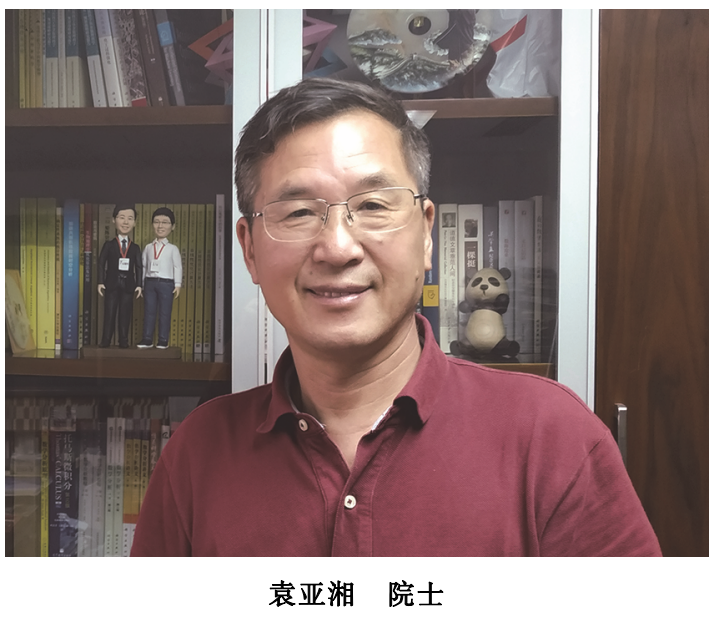

没有进展, 我的研究结果仍然是该问题世界上最好的. 这 是我最早在《中国科学》发表的文章, 是我做的比较重要 的工作之一.

您在《中国科学》发表的文章中, 有哪几篇是您印 象比较深刻的?

袁亚湘：这篇DFP算法收玫性的文章是我印象非常深 刻的. 虽然该文引用率不是很高, 但这个结果比较重要. 这个问题要得到彻底解决还很难, 希望将来能由我们中国 人推动这个问题的最终解决.

后来我和戴或虹合作, 我们对共轭梯度法研究影响力 比较大的是“戴-袁方法”, 这方面的系列工作多次发表在 《中国科学》, 所以印象也比较深刻.

另一个印象比较深的是, 2000年左右我和聂家旺合作 在《中国科学》发表的关于扩充的半定规划(SDP)问题的 文章“A potential reduction algorithm for an extended SDP problem”. SDP 是那时的研究热点, 是一个凸优化. 当时研 究的SDP都是矩阵变量的线性问题. 我和聂家旺在世界上 最早研究所谓扩充的SDP, 我们在《中国科学》发表的这 篇文章是国际上研究二次半定规划问题最早的工作, 所以 这篇文章印象也比较深. 
在您的众多成果中, 您最欣赏哪几项工作?

袁亚湘: 我自己比较得意的工作,一是关于信赖域的 系统研究; 二是共轭梯度法, 部分文章发表在《中国科学》; 三是拟 Newton 法, 证明了 BFGS 到 DFP(不含 DFP) 的 Broyden凸族的全局收玫性, 关于DFP的重要进展也发表 在《中国科学》; 四是子空间方法, 在国际数学家大会上 对该研究工作作过45分钟邀请报告. 我在这四方面做得比 较不错, 并且得到国际同行的认可.

\section{从编委到主编，履职尽责推动刊物不断提升}

您是如何成为《中国科学》编委的? 初任编委时, 对 《中国科学》的定位是如何考虑的, 对编委职责的预期 是怎样的?

袁亚湘: 我在《中国科学》发表第一篇文章之后的 1996 年, 成为《中国科学》编委. 那时, 《中国科学》还是一个 各个学科都在一起的大编委会, 杨乐先生是负责数学学科 的副主编. 在 20 世纪 90 年代中期, 我正好是中青年一代数 学工作者中在计算数学领域比较有代表性的一员, 杨先生 在1996年编委会换届的时候邀请我担任编委. 杨先生那时 定位《中国科学》是要办成中国数学最好的综合性刊物, 所 以一方面要求我们编委自己要投稿, 一方面要求编委审稿 时要严格把关. 从任编委之后直至2013年任主编之前, 我 投了不少文章, 包括后来共轭梯度法的系列文章以及我和 聂家旺的文章. 在审稿方面, 因为《中国科学》以纯数学 为主, 所以我任编委处理的稿件不是很多, 但我把关很严 格, 大部分投稿都被拒掉了. 从我任编委开始到现在二十 多年, 《中国科学》编委的职责变化非常大.
您从1996年9月起担任《中国科学》的编委, 2013年 1月起担任《中国科学: 数学》主编至今, 在这些年中, 《中国科学》发生了哪些令您印象深刻的变化?

袁亚湘: 我觉得最明显的变化是编委的参与度. 过去 杨乐先生作为主编付出很多, 但是那时编委付出的不是很 多, 现在编委的参与度与以前相比有极大的提高. 我任主 编之后, 调整了编委会结构, 增设了五位副主编, 这也符 合综合性期刊的特点, 对刊物发展有本质的影响. 虽然称 作副主编, 但实际上他们的很多权限跟主编完全一样, 与 主编一样有很高的责任感、热情和积极性参与期刊的工作. 我们每年召开全体编委会, 凝聚群体力量, 奖励优秀编委, 激励编委积极为期刊投人. 几位副主编积极参与, 编委参 与度极大地提高, 有更多的人把精力放在《中国科学》上, 这个变化非常明显.

第二个变化是作者的参与度. 一方面我们邀请了很多 优秀的稿件, 如开辟 “国际名家专栏” 邀请国内外著名数学 家投稿, 以及组织了一系列高水平的中英文专辑, 使得刊 物的学术质量不断提高. 另一方面我们采取了一系列包括 奖励优秀论文、奖励作者等做法, 近几年我们还定期举办 数学与交叉学科的高端学术论坛, 使得数学界更多优秀的 作者愿意积极地投稿, 这也是一个积极的变化.

第三是编辑部的变化. 首先, 现在编辑部的人员, 大 多是有数学背景的博士, 这与过去完全不一样. 其次, 过 去编辑们花在案头上的工作多一些, 现在的编辑更多地拜 访专家学者、参加国际国内学术会议, 更主动地参与学术 活动和组稿约稿.

当然, 这些变化发展取决于科学出版社的重视、中

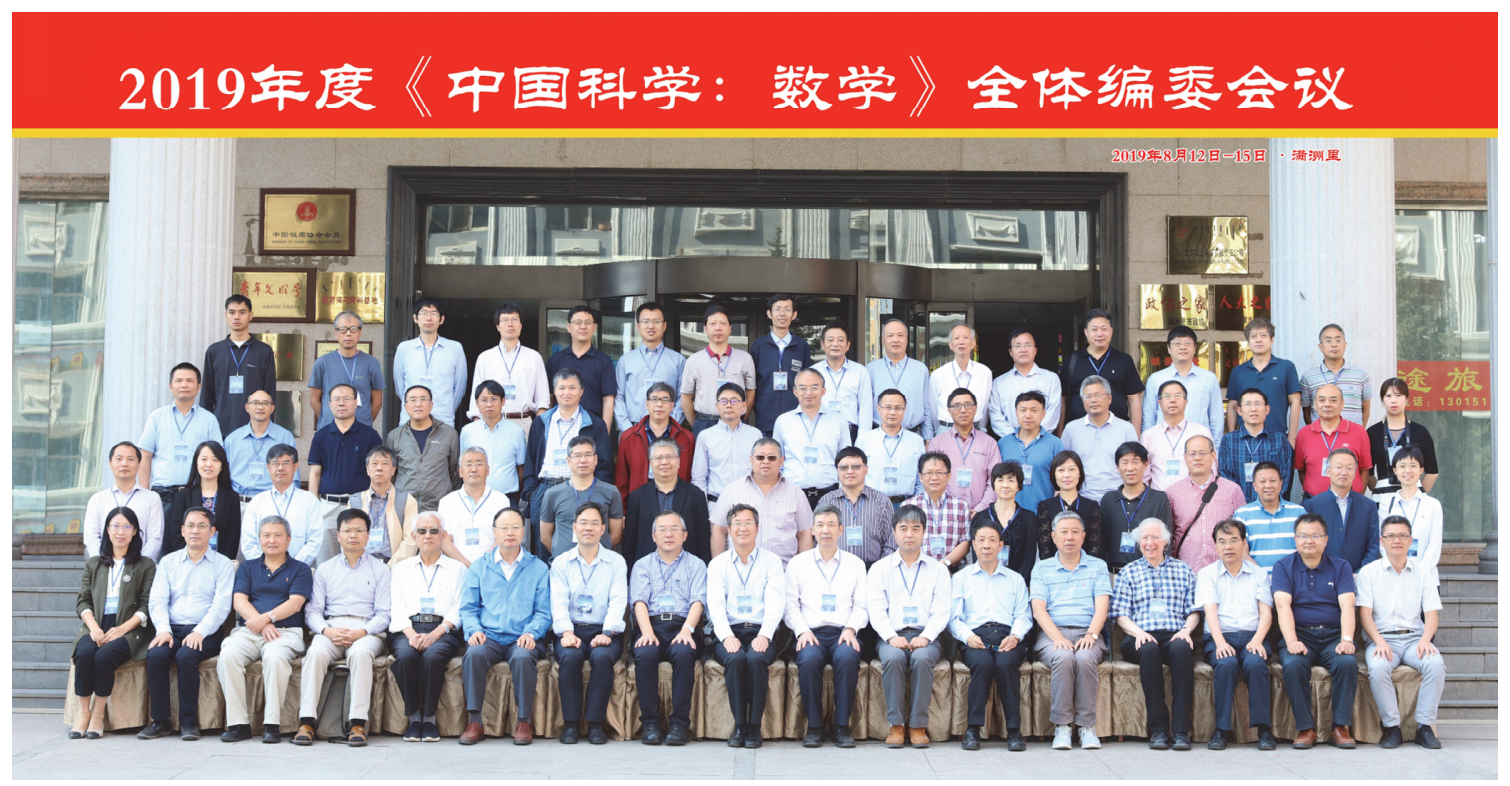

2019年度《中国科学: 数学》全体编委会议合影 
国科学院学部和国家自然科学基金委员会的重视, 以及中 国科学技术协会给我们的大力支持. 在此基础上我们推动 了一系列新举措, 使得刊物发生了很多积极的变化, 院士 投稿、杰出数学家和优秀的青年数学家投稿越来越多.

作为主编, 您觉得哪些情况最让您苦恼? 哪些事情 又能给您成就感?

袁亚湘: 我的主要烦恼就是, 中国作为数学大国向数 学强国迈进, 理应有与之相适应的数学强刊, 而我们刊物 距离成为国际一流强刊的目标还有很大差距.

作为主编, 我对刊物的期望是在学术上不断提高, 不 断挖掘优秀稿源, 发表优秀科研成果, 目标是办好期刊. 但是《中国科学》杂志社毕竟是企业, 还要面临经营和效 益的压力. 杂志社和编委会办刊的目标可能并不完全相 同, 这也是我苦恼的地方. 当然这是体制的原因, 在这方 面只能尽力“求同”.

谈到成就感, 第一, 很高兴看到数学界的同行对《中 国科学: 数学》认可程度越来越高. 实际上期刊好坏与否, 同行的认可最重要. 第二, 我任主编这七八年的时间, 越 来越多的编委也重视期刊, 愿意为她付出和负责. 第三, 越来越多优秀的数学家愿意把比较好的研究工作投稿《中 国科学: 数学》, 包括陆启铿院士、马志明院士、彭实戈 院士、田刚院士、张伟平院士、周向宇院士等. 第四, 在 国际上, 现在已经有非常热心的国际编委, 比如Arthur Jaffe愿意为期刊花费很多时间和精力. 这预示着一个很好 的开端, 我们希望有更多的国际编委能够积极参与咱们期 刊的建设. 这些都说明自己的付出, 得到了越来越多人的 认可.

您如何评价现在的《中国科学：数学》? 与您了解 的其他国内外期刊相比, 《中国科学: 数学》有哪些特 点和不足?

袁亚湘: 《中国科学: 数学》是一个处于上升阶段的 比较好的刊物. 我们以中国最好的数学刊物为起点, 以国 际最好期刊为目标, 正处于慢慢追赶的进程中, 虽然目前 与国际上最好的刊物, 比如数学的 “四大期刊”相比, 还有 很大的差距, 但是我们在不断进步.

经过多年的努力, 很高兴看到, 在数学界有越来越多 的人认可 《中国科学: 数学》, 很多著名的学者愿意把最好 的研究结果投来. 在国内, 《中国科学: 数学》已经成为大 部分数学家心目中向国内期刊投稿时的首选. 在国际上, 如果不说具体哪个专业领域, 不少国外数学家也认为 《中 国科学: 数学》是中国数学界最好的期刊. 总的来说, 无论 是期刊的学术声誉, 还是具体的办刊水平, 国内外越来越 多的数学家, 认为我们刊物是一个不错的数学期刊.

《中国科学: 数学》毕竟是综合性刊物, 与那些专业 性期刊相比, 有不足的地方. 比如在计算数学领域, 我们 国家研究计算数学的学者可能首先想到在国际上会投
SIAM(Society for Industrial and Applied Mathematics，美国 工业与应用数学学会)的期刊、国内投计算数学的专业期刊 $\mathrm{JCM}$ (Journal of Computational Mathematics). 所以, 《中国 科学: 数学》与那些专业性的期刊去竞争专业领域最前沿、 最热点的文章, 自然会有难度. 针对那些方向, 我们也许 可以更多地去邀请一些综述性文章, 介绍一些新学科, 开 创一些新方向, 朝这些方面去努力.

\section{科学家担当，呼吁改革数学教育 和加强文化自信}

您如何评价中国目前的数学教育和数学研究状况?

袁亚湘: 中国目前的数学教育, 很大的问题就是应试 教育和竞争过早, 这两个问题是相关的. 数学研究最重要 的是兴趣, 做得好与不好, 有没有兴趣很重要. 我们国家 的教育表面上可能大家认为是高等教育没抓好, 但实际上 根源是在中小学. 我们的中小学把分数看得非常重, 使得 大量青少年要做很多题, 孩子们慢慢就失去了对数学的兴 趣. 应试教育问题很大, 小学阶段有很多考试, 初中升高 中要中考, 高中升大学要高考, 在孩子小的时候竞争就非 常激烈, 导致孩子到大学竞争意识就弱了. 就数学研究而 言, 实际上在中小学学到的数学是“埃普西隆” (数学符号 $\varepsilon$, 指很小的量), 没有太多内容, 真正要做数学研究, 需要在 大学阶段把基础打好, 后面才能做好研究. 现在我们国家 正好相反, 高中之前数学那么一点点的“埃普西隆”, 大家 花了无穷大的精力去学; 到大学以后有无穷大的内容, 孩 子基本就花“埃普西隆”的精力去学了, 那根本学不好, 这 是很大的问题. 过早的竞争, 相当于一个长跑运动员, 前 面五公里跑得气喘吁吁, 跑到后面就跑不动了. 所以我们 国家的数学教育也好、数学研究状况也好, 应试教育和竞 争过早是根本的问题. 希望能有这样一个机制, 让我们的 青少年, 在小时候能每天快快乐乐, 没有太多压力, 到上 大学以后拼命学, 那就好办了.

近日, 《关于深化改革培育世界一流科技期刊的意 见》给中国期刊定出了明确的发展时间表: 到2035年, 我国科技期刊综合实力跃居世界第一方阵. 您认为要 实现这一目标, 迫切需要解决的问题是什么?

袁亚湘: 我觉得最重要的还是自信问题. 我们国家的 科技在进步，科技期刊也在进步，期刊的进步可能稍微慢 一些, 中国有很多一流科学家, 大家把大部分优秀的研究 工作都投到国际期刊了. 我观察到的现象是，中国目前很 多科学家把最好文章的三分之一、甚至四分之一投到中国的 期刊, 把四分之三投到国外期刊. 美国科学家肯定有三分 之二以上最好的文章投在美国的期刊, 欧洲科学家可能也 有一半左右的文章投到自己国家的刊物, 这是自信的表现. 如果中国科技将来稳居世界第一方阵，那么中国的科技期 刊肯定也应该位居世界第一方阵. 所以说, 要办好中国的 
科技期刊, 最迫切的问题是自信. 中国自己的科学家首先 要把最好的文章, 至少一半以上, 投到中国自己的期刊.

第二是评价体系的问题. 现在评价科研工作还是过分 看重文章发表在什么期刊, 工作本身却显得不是那么重 要. 所以我鼓励, 在科技评价的时候, 我们不要谈期刊, 而只谈具体工作, 这个工作发表到哪个期刊都一样. 比如 冯康先生关于有限元的工作, 没有人关注有限元的文章发 表在什么期刊; 冯康先生的辛算法获得了国家自然科学一 等奖, 他关于该方向上的所有文章都发表在国内的期刊 上. 靠期刊来提高自己的声望, 是在搭别人的车, 而没有 体现工作本身的重要性.

再一个要解决的问题是不要太急功近利. 还是拿跑步 来比喻, 假设我们都是长跑运动员, 现在美国期刊和欧洲 期刊在第一梯队, 我们中国期刊在第二梯队. 我们要明白, 只要自己有加速度, 能够有劲在跑, 沉下心来坚持跑, 就 一定会追上去. 而不是跑两步就抬头看一下, 数一数我是 第几名, 还没跑几步呢又数一下我是第几名, 那当然越来 越跑不动. 真正想要将来达到目标, 就是要沉住心, 明确 目标, 知道该怎么做, 好好坚持去做. 比如我们先把国内 最优秀学者的文章, 至少争取一半发表在我们期刊, 国际 上也吸引一些好文章, 坚持这么做, 慢慢我们就变成好期 刊, 要有这个信心.

\section{放眼未来, 寄语刊物长久发展}

对《中国科学: 数学》的未来发展, 您有哪些建议 与期望?
袁亚湘: 第一个建议, 《中国科学: 数学》虽然是综合 性的数学刊物, 但在学科布局方面还是要以基础数学为 主, 无论是发表文章的学科分布, 还是学术发展方向, 应 该向国际数学界公认的顶尖数学期刊靠拢. 我们要了解 Annals of Mathematics 等“四大期刊”发表文章的学科分布, 向它们看齐, 也要关注国际上最前沿、重要的、活跃的研 究方向. 通过发表文章, 慢慢引导国内数学界真正瞄准国 际前沿的方向去开展研究工作, 这也是一个刊物很重要的 职能. 当然, 我们期刊也应吸引优秀的、真正有原创思想 的文章, 成为一个鼓励中国数学家勇于闯新路、敢于提出 自己的新的数学问题、开辟新的数学研究方向、推动数学 发展的重要学术交流平台.

第二个建议, 我们目前的重心和工作重点, 还是要吸 引国内最好的工作. 着眼国际最好的成果, 吸引国际上的 好文章, 这是我们希望做到的. 但《中国科学：数学》毕竟 是中国人办的刊物, 主要依托和依靠的对象还是国内数学 家, 所以重点还是要尽可能多地吸引国内最好的工作, 这 是我们真正的根本. 做到了这一点, 我们才能进一步去吸 引国际上最好的工作. 这也要求刊物主编、副主编、编委、 编辑部, 要把握学术前沿, 要了解国内有哪些学者的工作 做得非常好, 能够主动跟他们建立好联系; 要了解有哪些 国内的重要研究, 近期有可能会有突破; 要关注国内中青 年数学家, 关注他们做的真正好的工作, 想办法把最好的 工作，吸引到我们刊物来.

期望《中国科学: 数学》迈向国际化、成为国际一流 期刊的一天早日到来. 